\title{
Alte und neue Medialität an der Schwelle zur digitalen Wende. Das mediale Echo Thomas Bernhards und die Selbstinszenierung Elfriede Jelineks
}

Old and new mediality on the threshold of the digital revolution. The media echo of Thomas Bernhard and the self-staging of Elfriede Jelinek

Zdeněk Pecka

\begin{abstract}
The Austrian authors Thomas Bernhard and Elfriede Jelinek established themselves as personalities whose radicalism, provocations and public gestures became part of the impact of their work. It can be seen that the radical reflections of the narrators and characters are a statement continuation of the authors. At Bernhard, the ways which communicate this self-staging are still "old" (press and television). Jelinek exclusively uses the "new" medium Internet and the website already set up at the beginning of the digital turn for her self-presentation and publication.
\end{abstract}

\section{Keywords}

Thomas Bernhard; Elfriede Jelinek; Austrian literature; self-staging; digital turn; media; new media; authorship 
„Aber schreiben und lesen muß man dafür schon können. Das ist das Minimum. Diese Kulturtechniken - niemals waren sie so wertvoll wie heute. So, und warum kommen dann von hier, wo ich bin und gleichzeitig auch Sie sein können, immer nur (und schon wieder) negative Meldungen, und von Ihnen auch, Sie Autorin Sie, Nichtmitglied der Auto- Rennversammlung, nein, Rinnenversammlung?" 1

Das aus dem Kontext der assoziativen Erzählerrede herausgenommene Zitat aus dem Internet-Roman „Neid“ von Elfriede Jelinek soll zeigen, wie fraglich manche theoretischen Aspekte der Autorschaft in der neuesten digitalen Literatur werden können. Das schwankende (Nicht)Unterscheiden der Kategorien Autor/in-Erzähler/in-Figur kann die Autorin im Barthes'schen Sinne ${ }^{2}$ aus der Interpretation des Romans verabschieden, wobei sie jedoch alle rhetorischen und erzählerischen Mittel beibehält, um die äußere Wirklichkeit, traumatisierende historische Ereignisse sowie z.B. ein eigenes Unrecht aus dem Text hinaus kommentieren zu können. Im Vortrag von Michel Foucault, der auf die Überlegung von Barthes zurückgreift, steht unter anderem:

„[...] ein Autorname ist nicht einfach ein Element in einem Diskurs [...]; er hat bezogen auf den Diskurs eine bestimmte Rolle: er besitzt klassifikatorische Funktion; mit einem solchen Namen kann man eine gewisse Zahl von Texten gruppieren, sie abgrenzen, einige ausschließen, sie anderen gegenüberstellen. Außerdem bewirkt er eine Inbezugsetzung der Texte zueinander. [...] Hat ein Diskurs einen Autorennamen, kann man sagen, »das da ist von dem da geschrieben« oder »ein gewisser ist der Autor von...«, so besagt dies, daß dieser Diskurs nicht aus alltäglichen, gleichgültigen Worten besteht, nicht aus Worten, die vergehen, vorbeitreiben, vorüberziehen, nicht aus unmittelbar konsumierbaren Worten, sondern aus Worten, die in bestimmter Weise rezipiert werden und in einer gegebenen Kultur ein bestimmtes Statut erhalten müssen.“3

Die Diskursivität der Autorennamen Thomas Bernhard und Elfriede Jelinek wird gerade in der Dichotomie des Öffentlichen und Privaten - in der Grenzüberschreitung der Fiktionalität und Authentizität eines literarischen Textes deutlich. Die Prinzipien der komplizierten und nicht nur streng medial bedingten Kommunikation sind Selektion, Schaffung von Verständlichkeit, Strukturierung des Wissens sowie Begründung und Transparentmachung des Transfers. Dies gilt einerseits für die Kommunikation unter Experten, bei Thomas Bernhard im Bereich der Literaturwissenschaft und der Germanistik. Gleichen Kriterien in anderen Formen unterliegt andererseits die Experten-Laien-Kommunikation, in der vermittelnde Laien auftreten - gemeint sind journalistische Felder der GateKeeper und Journalisten, in denen das Wissen sortiert und durch die es kommuniziert wird. Bereiche der Bildungssphäre, Doktoranden und Studierende, die ihre Abschlussarbeiten schreiben, Institutionen, Schulen und Lehrende, die über ihre

1 JELINEK 2007-2008, S. 91.

2 Vgl. hierzu BARTHES 2000, S. 185-197.

3 FOUCAULT 2000, S. 210. 
Lektüre-Kanons entscheiden, sowie Grenzfelder, auf denen z. B. Mediatoren begleitende literarische Events oder Laien ihre Lesezirkel veranstalten, sollen auch nicht vergessen Werden. Die zentrale Bedingung der Vermittlung sind die Medienspezifika und ihre Darstellungsformen. Gerd Antos unterscheidet im Wesentlichen 1. das sprachlich-textuell konstituierte oder repräsentierte Wissen, 2. das bildlich und graphisch konstituierte oder repräsentierte Wissen, 3. das durch formale Sprachen wie Mathematik, Statistik oder Logik konstituierte oder repräsentierte Wissen, und 4. das Wissen in digitalen Konstitutions- und Repräsentationsformen (Multimedia). ${ }^{4}$ Die (Selbst-)Darstellung in den Konstitutionen 1 und 4 ist das Thema des vorliegenden Artikels. Es sollen auffallende Formen der medialen (Selbst)Präsentation, von den Zeiten der Selbstinszenierung Thomas Bernhards bis zum annoncierten Rücktritt ins Private Elfriede Jelineks ${ }^{5}$ in der Epoche der beginnenden digitalen Wende ${ }^{6}$ gezeigt werden.

\section{Autor, Erzähler, Mediator}

Eine unumstrittene Regel der gegenwärtigen medialen Kommunikation sagt, dass öffentlich auftretende Persönlichkeiten in Zeiten der zersplitterten und unstabilen medialen Wirklichkeit an ihrem medialen Bild ständig arbeiten sollen. Die Prinzipien des medialen Self-Marketings gelten nicht nur in besonders exponierten Bereichen des Konsums und der Pop-Kultur. In der Kunst also auch in der Literatur ist das mediale Echo (nicht nur in der Literaturkritik) mindestens seit der Aufklärung ${ }^{7}$ ein wichtiger Indikator des Ruhmes und Ansehens. Eines der effektivsten Mittel der medialen Kommunikation im Bereich der modernen Literatur sind Innovation und Nichtkonformität, die häufig bis zu skandalhaften Ereignissen führen. Neben den teils schon erwähnten Theorien der Autorschaft muss hier noch nach Bourdieus literatursoziologischen Theorien gegriffen werden, denn Bourdieu beobachtet, dass die Nichtkonformität in den Feldern der Kunst und Literatur kurzfristig keine ökonomischen, sondern symbolische Profite in Form von Akzeptanz in intellektuellen Kreisen bringt. Manchmal können sie auch als Investition verstanden werden, die später zur Durchsetzung führt und zum ökonomischen Profit wird. Der auf diese Weise etablierte Autor hat den Weg zum Ruhm und dadurch auch zu einigen Formen des literarischen Kanons bereits betreten (und wird allmählich selber zu jemandem, von dem sich wieder andere Avantgardisten abgrenzen müssen). ${ }^{8}$ Dies ist das Feld, in dem sich Thomas Bernhard nicht nur als innovativer Autor, sondern auch als ein aktiver Mitgestalter des Bernhard'schen Diskurses in den Medien bewegte. Seine

4 ANTOS 2005.

5 S. JELINEK 2004.

6 „Die Entwicklung der digitalen Medien und die Etablierung des Internet als neues Leitmedium haben die herrschenden Kulturformen bereits grundlegend verändert und werden damit auch in Zukunft fortfahren." SIMANOWSKI 2002, S. 9.

7 Vgl. hierzu HOHENDAHL 1985.

8 BOURDIEU 2001, S. 405. 
Figuren provozieren, weil Bernhard bewusst mit der Voraussetzung arbeitete, dass die Rezipienten ungenügend zwischen dem Autor und seinen Erzählern unterscheiden.

Neben den traditionellen Printmedien und dem Fernsehen kommen nach der Ära Thomas Bernhards noch die neuen Medien hinzu, die die mediale Landschaft sowie das Verhalten der Leser, Konsumenten, schließlich auch Surfer und nicht zuletzt die Selbstpräsentation der Autoren markant verändert haben. Matthias Jung fasst den Wandel im Verhalten der Leser zusammen, wenn er über den Wissenstransfer durch neue Medien schreibt:

„Das Bild des Surfers, der oberflächlich über den Text hinweggleitet bzw. gefördert durch Hyperlinks im Text hin- und herspringt, dürfte auch bei Printmedien weitgehend der Realität des Freizeitlesens entsprechen. ${ }^{“ 9}$

Methoden der Transferwissenschaften und Digital Humanities beschreiben die komplizierten Wege des schriftlich festgehaltenen und schließlich digitalisierten Wissens oder Denkens vom Autor oder Verfasser zum Rezipienten, sodass die von der Literaturwissenschaft traditionell betrachteten Praktiken der Produktion, Rezeption, Vermittlung und des Transfers von Literatur durch die Digital Humanities aktualisiert werden müssen.

\section{Das alte mediale Echo bei Thomas Bernhard}

Laut Foucault geschieht die Konstruktion eines Autorenbildes diskursiv, ist, vereinfacht formuliert, zum Teil ähnlich wie bei Bourdieu, besonders von den institutionellen Machtverhältnissen abhängig und unterliegt zugleich dem jeweiligen gesellschaftlichen sowie historischen Kontext. ${ }^{10}$ Im Rahmen dieser Gesetzlichkeiten wird auch die Selbstvermarktung Thomas Bernhards deutlich. Der österreichische Schriftsteller und Dramatiker arbeitete sehr zielstrebig an seiner öffentlichen Pose und an seinem medialen Bild. Dies ist schließlich zu seinem qualitativen Merkmal geworden. Der Autor ist dann fester Bestandteil seiner Werke, denn Bernhards öffentliche Gesten wurden allmählich zu begleitenden Elementen seiner Veröffentlichungen oder der (Ur-)Aufführungen seiner Theaterstücke. Wendelin Schmidt-Dengler benennt das Phänomen in seiner bekannten These: Bernhards Werk sei die „[...] Wirkung als eigene Qualität zugewachsen und von diesem nicht mehr zu trennen [...]." ${ }^{\text {"1 }}$ Die Rezeption von Bernhards Spätwerk zu Lebzeiten wäre ohne ein gewisses Vorwissen über sein mediales Bild unmöglich gewesen. Es lassen sich jedoch bestimmte Aspekte der Medialität beobachten, die seinen Ruhm und seine Kanonisierung auch nach seinem Tod im Februar 1989 geprägt haben. Im weiteren Sinne lässt sich von einem sehr anschaulichen posthumen medialen Literatur-Transfer sprechen. Der Prozess, in dem Thomas Bernhard zum festen Bestandteil des Kanons der österreichischen Literatur wurde, lässt sich mit Bourdieu als eine Art Landkarte

9 JUNG 2001, S. 282.

10 Vgl. hierzu FOUCAULT 1997.

11 SCHMIDT-DENGLER 1989, S. 107. 
zeigen, ${ }^{12}$ auf der einige Wege der Vermittlung beschrieben werden. Bei der Unterscheidung des individuellen und kollektiven literarischen Kanons muss auf das Feld der zeitgenössischen professionellen Leser aufmerksam gemacht werden. Kritiker, Theatermacher und Theaterwissenschaftler, Germanisten, Literaturwissenschaftler, Verleger, Übersetzer oder einflussreiche Intellektuelle, die gemeinsam hatten, Thomas Bernhard und seine Werke zu schätzen, konstituierten in der Bernhard'schen literarischen Landschaft ein relativ homogenes literarisches Feld. In den vergangenen Jahrzehnten haben sie Bernhards Prosa und Dramen als kanonfähige Texte anerkannt und in den Netzwerken der Gattungstradition und intertextuellen Verweise fest verankert. Besonders die Literatur- und Theaterwissenschaft hat die Bernhard'schen Stoffe und Motive, die direkten sowie indirekten Bezugnahmen auf andere Texte sowie andere unumstrittene literarische Qualitäten beschrieben, kategorisiert und kontextualisiert. Bernhards Texte haben damit den persönlichen, individuellen Kanon der Leser überragt und sind zum offiziellen Kanon, zur schulischen Muss-Lektüre nicht nur in Österreich, sondern auch im Ausland geworden. ${ }^{13}$

Die Etablierung des Werkes von Thomas Bernhard im Kanon der österreichischen bzw. europäischen Literatur erfolgte jedoch auf mehreren Machtfeldern im Sinne von Bourdieu und Foucault. Der Transfer verlief auf textuellen, bildlichen und natürlich auch multimedialen Wegen im Sinne von Antos. ${ }^{14}$ Auf der Landkarte der Vermittlung sind Machtfelder nicht nur der Germanistik und Literaturwissenschaft, der Literaturund Theaterkritik, sondern auch des Kulturbetriebes sowie der Macht des Staates, der Bundesländer sowie regionaler oder kommunaler Verwaltungen zu finden, des weiteren institutionelle Machtfelder der Verlage und Vereine usw. Die einzelnen Felder beeinflussten sich gegenseitig und unterschiedlich im Verlauf der Zeit, jedoch sind auch herausragende Ereignisse und Prozesse zu beobachten, die über wesentlich mehr Einfluss als andere verfügten. Prägend und in der Sekundärliteratur ausreichend beschrieben sind die erregten Rezeptionsgeschichten der Werke „Holzfällen“ und „Alte Meister“ oder „Auslöschung“, die heftige Debatten nicht nur in Österreich, sondern auch im Ausland hervorriefen. Die Buchexemplare von „Holzfällen“ wurden im Oktober 1984 beschlagnahmt, denn der frühere Freund Bernhards und Komponist Lampersberg fühlte sich durch die Figuren des Ehepaars Auersberger öffentlich denunziert und lächerlich gemacht. ${ }^{15}$ Das im Auftrag des Burgtheaters entstandene Drama „Heldenplatz“ wurde 1988 in dem Bedenkjahr (50 Jahre Anschluss, 100-jähriges Bestehen des Wiener Burgtheaters) unter der Regie von Claus Peymann im Wiener Burgtheater uraufgeführt und die Vorstellung sowie ihre Vorbereitung davor wurden zum medialen Skandal. Scheltreden, Hasstexte, Manipulationen v.a. in den Printmedien oder Denunziationen begleiteten die Vorbereitung der Uraufführung im öffentlichen und medialen Raum. ${ }^{16}$ Die Konsequenz war das testamentarische

12 Mit der Metapher einer literarischen Landschaft - sogar eines literarischen Kontinents - spielt auch der umfangreiche Band über die ausländische Rezeption des Werkes von Thomas Bernhard. S. BAYER 1995.

13 Vgl. hierzu PECKA 2010, S. 107-116.

14 ANTOS 2005 [Anm. 4].

15 Vgl. hierzu z.B. LINK 2000.

16 Vgl. hierzu BURGTHEATER WIEN 1989. 
Verbot aller Formen der Publikation von Werken Thomas Bernhards, das erst durch die Thomas-Bernhard-Privatstiftung 1998 umgangen werden konnte.

Das posthume literarische wie auch menschliche Bild des Autors beeinflusste neben anderen Ereignissen (Ausstellungen des Thomas-Bernhard-Archivs, Lesungen usw.) die Herausgabe der Korrespondenz Thomas Bernhards mit Siegfried Unseld in einem Sonderband des Suhrkamp Verlags. ${ }^{17} 524$ Briefe, Telegramme und Kurzmitteilungen umfasst der Band und wurde dadurch zur Chronik einer besonderen Beziehung. Der veröffentlichte Briefwechsel enthüllte den Schriftsteller, wie er früher eher weniger bekannt gewesen war. In der Korrespondenz werden künstlerische oder literarische Themen kaum besprochen, fast immer geht es um Geld und Aufmerksamkeit. Bernhard fühlte sich vom Suhrkamp Verlag ungenügend behandelt. Am 24. November 1988 schickte Unseld sein letztes Telegramm, am folgenden Tag schrieb Bernhard zurück:

„Wenn Sie, wie Ihr Telegramm lautet, ,nicht mehr können“, dann streichen Sie mich aus Ihrem Verlag und aus Ihrem Gedächtnis. Ich war sicher einer der unkompliziertesten Autoren, die sie jemals gehabt haben." ${ }^{18}$

Einen Monat später trafen sich die beiden zur Versöhnung. Vierzehn Tage darauf, am 12. Februar 1989, starb Thomas Bernhard. Posthum, im Jahre 2009, wurde ein anderer Text Bernhards herausgegeben - die Prosasammlung „Meine Preise“. ${ }^{19}$ Das Werk kommt aus dem Nachlass, der im Frankfurter Suhrkamp Verlag systematisch herausgegeben worden ist. Der Text wurde als noch nicht veröffentlichte Handschrift entdeckt und der Verleger hat sich für die Herausgabe entschieden, jedoch außerhalb des gesammelten Werkes $^{20}$. Die fehlende Redaktionsarbeit ist an manchen Kapiteln oder Abschnitten des Textes zu erkennen, man muss jedoch davon ausgehen, dass „Meine Preise“ nun ein Zeugnis aus dem Nachlass und keine ambitiöse Belletristik ist. Der sehr private Text weist viele Gemeinsamkeiten mit Bernhards autobiografischer Prosa auf. Der Unterschied zwischen Autor und Erzähler verwischt sich, was von den Herausgebern auch noch durch Eingliederung einzelner Dankesreden in den Dokumentationsteil unterstrichen wurde. Das Buch ermöglicht einen Blick hinter die Pose des Autors in das Erlebnis eines professionellen literarischen Werdegangs.

\section{Die neue Medialität und Elfriede Jelinek}

In Verbindung mit den modernen Medien, die Bewegung in das als starr gedachte Gattungsschema brachten, ermöglicht der zunächst als literarischer Blog entstandene

\footnotetext{
17 FELLINGER 2009.

18 FELLINGER [Anm. 17], S. 806.

19 BERNHARD 2009.

20 Vgl. Dazu Anm. 19, s. Buchumschlag.
} 
Internet-Roman Elfriede Jelineks „Neid“21 eine neue Betrachtung der schriftstellerischen Geste, mit der man intertextuelle Bezüge herstellt, die Mentalität des Volkes sowie die Politik kritisiert und eine neue Form der österreichischen Anti-Heimatliteratur definiert. ${ }^{22}$ $\mathrm{Zu}$ diesen Aspekten tritt auch die veränderte Form der Rezeption von digitaler Literatur, bzw. der Erwartungshorizont der Leser oder Konsumenten der digitalen Medien, für die der Text vom Anfang an bestimmt war. ${ }^{23}$ Alles zusammen bildet ein dichtes Gewebe von Bedeutungen und Anspielungen, das zu einer wirksamen Selbstpräsentation der Autorin wurde, besonders nachdem sie im Interview für die österreichische Zeitschrift „Profil“ ihren Rückzug aus dem öffentlichen Raum angekündigt hatte. ${ }^{24}$ Mit der Einrichtung der Internet-Präsentation auf der Website der Autorin 1996 hielt Elfriede Jelinek Schritt mit dem Fortgang der digitalen Wende im deutschsprachigen literarischen Feld. Allmählich überschritt sie auch die Grenze des Beiwerks, des Epitextes, ${ }^{25}$ denn die Website liefert seitdem nicht nur ergänzende Informationen über Biographie, Veranstaltungen u. ä. Lena Lang betont in ihrer Analyse der Website von Jelinek:

„Elfriede Jelinek [...] nutzt ihre Website schon seit der Erstellung im Jahr 1996 primär als Publikationsort für literarische Texte [...]. Sie veröffentlicht hier bis heute Prosa- und Theatertexte, Notizen und Essays zu gesellschaftspolitischen und kulturellen Themen, zur eigenen Biographie und Poetologie, Nachreden, Preisreden und weitere Textsorten. ${ }^{26}$

Die Präsenz der Autorin sowie ihrer Texte im Internet illustriert die Veränderung der oben am Beispiel Thomas Bernhards beschriebenen Wege des Transports von Literatur und die Rolle des Autors. Mitte der 90er Jahre war eine Zeit, die sich von der digitalisierten Literatur noch ziemlich viel versprach. Es entstand das heute schon eingestellte Projekt „Netzliteratur.de“, in dem sich deutschsprachige Künstler, Autoren oder Akademiker vernetzten. Später hat an dieses Projekt die Vernetzung Netzliteratur.net

\section{JELINEK 2007-2008.}

22 Eine der ersten Definitionen der sog. Anti-Heimatliteratur in Österreich der späten 60er und 70er Jahre liefert Jürgen Koppensteiner 1982: „Besonders in Österreich wurde in den siebziger Jahren Heimat immer wieder thematisiert. Diese neue Heimatliteratur hat aber nichts mit dem zu tun, was man gemeinhin unter Heimatliteratur versteht. Es geht nicht um die Darstellung einer heilen Welt und schon gar nicht um die einer irrealen Ersatzwelt à la Ganghofer, sondern um eine Heimatliteratur, die die Heimat, den ländlich-bäuerlichen Raum als kaputt denunziert. Diese neue Art von Heimatliteratur wurde als ,negative“ [sic], als ,problematische“ [sic], ,kritische‘ oder ,ironische‘ Heimatliteratur bezeichnet. Sehr schnell hat sich hierfür schließlich der Begriff Anti-Heimatliteratur eingebürgert und auch durchgesetzt.“ (KOPPENSTEINER 1982: 1)

23 Jelinek selbst hat es formal bestätigt, indem sie dem Roman eine Art „Nachwort“ hinzugefügt hat, in dem sie die ausschließlich elektronische Version des Textes rechtfertigt. „Dieser Text mit Namen Neid gehört nicht in ein Buch. Er gehört nicht auf Papier, er gehört in den Computer hinein, dort habe ich ihn hineingestellt, dort habe ich ihn deponiert, dort kann er in Ruhe verderben wie Müll (nur auf Wunsch und mit Hilfe einiger Knopfdrückereien können Sie ihn sich aber holen, wann Sie sollen, solang Sie und soviel davon wie Sie wollen), und bin dann einfach weggegangen. Ich weiß ja, daß der Roman dableibt, auch in meinem eigenen Gerät mit dem Flachschirm. Er ist zur Entnahme frei, der Text, was ich nicht bin." (JELINEK 2008)

24 JELINEK [Anm. 5].

25 Vgl. hierzu GENETTE 2001.

26 LANG 2016, S. 3. 
angeknüpft. ${ }^{27}$ Netzliteratur.de sollte ein Knoten sein, in dem man alle wichtigen Kontakte, theoretische Texte zur vernetzten Internetliteratur sowie eigene Experimente der Autoren findet.

„Dann gehören hierzu sicher auch die Webseiten der international bekannteren Autoren und Künstler wie Friedrich Block, Florian Cramer, Johannes Auer, Susanne Berkenheger, Frank Klötgen, Esther Hunziker, Jörg Piringer, ÜBERMORGEN, AND-OR und anderer. Schließlich sollten die Initiativen einzelner Forscher, Forschungsinstitute einbezogen werden. ${ }^{28}$

Beate Suter beschreibt in ihrer ausführlichen Studie die Geschichte der deutschsprachigen Netzliteratur von den anfänglichen Experimenten der Stuttgarter Gruppe im Jahr 1959 bis zum massiven Einstieg der social media in den 10er Jahren des 21. Jahrhunderts, die v.a die auf Hypertext basierte Literatur verdrängt haben. ${ }^{29}$ Für die zweite Hälfte der 90er Jahre sind v.a. Experimente typisch, die versuchen, der konkreten Poesie eine neue Dimension zu liefern.

„Konkrete elektronische Poesie in Perfektion bietet auch das Paradebeispiel worm applepie for döhl (1997) von Auer. Dieses animierte Gedicht basiert auf Döhls konkreter Inkunabel apfel (1965). In diesem Apfel ist sprichwörtlich der Wurm drin. Das animierte Poem worm applepie for döhl (1997) fügt dem ursprünglichen Werk einen spielerischen Aspekt hinzu [...].“30

Gleichzeitig entwickelten sich erste Versuche des kollaborativen Schreibens wie etwa die Projekte „Absolut Homer“ (1992), „Hyperknast“, „Hypertexttrees“ (beide 1995-1996), die erotische Geschichte „Beim Bäcker“ (1996) oder „taxis“ (1995). „Die 22 Autoren steuerten alle einen ihrer Texte bei. So ist ,taxis` zu einem Hypertextnetzwerk geworden, das aus dem von Walter Grond initiierten Literaturprojekt ,Absolut Homer“ entstand.“31 Eine wichtige Voraussetzung des Schreibens im Bereich der animierten computergestützten konkreten Poesie oder der auf Hypertext basierten Literatur war die Beherrschung des Programm-Codes und der tiefenstrukturspezifischen technischen Mittel. Diese Linie der digitalen Literatur zeigte sich jedoch allmählich als nicht überlebensfähig. ${ }^{32}$

27 AUER 2002-2012.

28 SUTER 2012, S. 4.

29 Den langsam verschwindenden zuvor aber in die verlinkte Literatur projizierten Optimismus repräsentiert z.B. der Beitrag des SWR vom 30. 4. 2013 „Die Zukunft des Lesens ist das Nicht-Lesen. Neue Medien, neue Literatur?" über die stattgefundene Litflow-Konferenz in Berlin. Im letzten Absatz muss die Autorin konstatieren: „Doch egal, wo man hinsieht: Alle lesen immer noch Bücher. Alle nehmen immer noch das gute alte gedruckte Buch in die Hand, auch wenn viele, vor allem die Jüngeren, mittlerweile mehrgleisig fahren. Und auch in gewisser Weise mehrgleisig denken: Digital und Analog. Im Buch und ums Buch herum. Aber alle lesen noch Bücher - das klingt wie die letzte Pointe der Litflow-Konferenz." MÜLLER 2013.

30 SUTER [Anm. 28], S. 9.

31 SUTER [Anm. 28], S. 15. Suter benennt gleichzeitig ein wichtiges Problem der Internetliteratur generell: „Das Projekt „taxis“ war von 1995 bis ca. 2006 im WWW zu finden. 2006 wurde das GEWI-Lab der Universität Graz geschlossen und die alten Daten verschwanden vom Server.“ (Ebenda).

32 Vgl. hierzu LORENZ 2013. 
Was aber der traditionellen Literatur am meisten ähnelt, ist die Produktion der Texte in literarischen Blogs. Die Genese wie auch Distribution der Texte ist zwar technisch bedingt, indem man Geräte nutzt, die imstande sind, den im Internet gespeicherten Inhalt abzubilden, herunterzuladen oder mittels anderer Tools zu durchsuchen, zu katalogisieren usw., jedoch folgen die Texte den alten und bekannten Wegen der Produktion, Distribution und Rezeption.

Den Beginn der deutschsprachigen blog-artigen Literatur setzt Suter mit Einschränkungen auch in die zweite Hälfte der 90er Jahre, es dürfte nicht falsch sein, „[...] mit dem Projekt Abfall für alle von Rainald Götz [!] von 1998 zu beginnen, eine Art öffentlich geführtes Tagebuch voll von flüchtigen Gedankenspritzern zum eigenen Alltag. “33 Hier führt schon eine direkte intertextuelle Verbindung zu Elfriede Jelineks Roman „Neid“ zurück, in dem im Untertitel seiner PDF-Version zu lesen ist: „(mein Abfall von allem)“. ${ }^{34}$ Es handelt sich um eine eindeutige Anspielung auf den literarischen Blog von Rainald Goetz, mit dem sich auch Jelinek programmatisch zu der deutschsprachigen Internet- und Blog-Literatur anmeldet. ${ }^{35}$ Somit zählt das auf der Homepage ${ }^{36}$ der Autorin publizierte Schaffen zum Feld der sekundären literarischen Kommunikation im Netz, nach Peer Trilckes „Literatursoziologie im Internet“:

„In den Blick gerät damit ein mittlerweile ebenso differenzierter wie formen- und sphärenreicher Phänomenkomplex: Er erstreckt sich, um nur einige Formen und Sphären zu nennen, von der professionalisierten Literaturkritik auf den Onlineportalen der klassischen Printmedien, der Autorenwebsite16 oder den On-linemarketing-Aktivitäten [!] der Verlage über genuine eMagazine, Foren, Blogs und Microblogs oder Portale wie Amazon, Lovelybooks, Goodreads, Youtube et cetera bis hin zu den unzähligen sekundären literarischen Kommunikationshandlungen auf Social Cataloging- und Social Networking-Plattformen.“" ${ }^{\text {“7 }}$

Dass sich Jelinek der Bedingtheit der maschinellen Distribution der Texte bewusst ist und dass sie sie programmatisch als Bestandteil der aktuellen Selbstpräsentation nimmt, beweist auch das Zitat aus „Keine Anweisung, keine Auszahlung, kein Betrag, kein Betrug. (Ein paar Anmerkungen zu Neid)“:

33 SUTER [Anm. 28], S. 30.

34 JELINEK [Anm. 21], S. 1.

35 Vgl. hierzu LANG [Anm. 26], S. 15.

36 „Die Erstellung der (literarischen) Website fällt genau in den Zeitraum, in dem sich Elfriede Jelinek zum ersten Mal aus der (österreichischen) Öffentlichkeit zurückgezogen hat, da die persönlichen Angriffe auf sie als ,Nestbeschmutzerin` einen Höhepunkt erreicht haben. Aufgrund der Ressentiments seitens der rechtspopulistischen Freiheitlichen Partei Österreichs (FPÖ) und der auflagenstarken österreichischen Boulevardtageszeitung Die Neue Kronen Zeitung (kurz Krone) sieht sie sich im Jahr 1995 dazu veranlasst, ein Aufführungsverbot ihrer Stücke im eigenen Land zu verhängen, das bis 1997 aufrechterhalten wird - ganz in der österreichischen Tradition Thomas Bernhards, der ein solches Verbot allerdings erst posthum verfügte." LANG [Anm. 26], S. 3.

37 TRILCKE 2013, S. 5-6. 
„Ich weiß ja, daß der Roman dableibt, auch in meinem eigenen Gerät mit dem Flachschirm. Er ist zur Entnahme frei, der Text, was ich nicht bin. Ich bin nicht frei, schon gar nicht zur Entnahme, wer würde mich auch nehmen, wer würde denn dem etwas entnehmen wollen, was ich sage? Ich hebe ja oft Tagesneuigkeiten und Aktualitäten, auch Klatsch und Tratsch, in die Texte hinein, um ihnen ihr Verfallsdatum einzuprägen. Das muß man ihnen immer wieder einbläuen, sonst vergessen sie es. Jeden Augenblick können sie fällig sein, und das ist gut so. Wenn ich sterbe, warum soll dann dieses Geschreibe leben dürfen? Es darf aber, irgendwo wird es überleben, in irgendeiner Maschine. Ich hätte vieles, das mir zu intim war, niemals in einem Buch schreiben wollen und können. Es soll so schnell verzehrt sein wie ein Hamburger oder eine Leberkässemmel. Es ist zum raschen Verbrauch bestimmt. “38

Der Roman selbst wurde in Form eines literarischen Blogs auf der Homepage Elfriede Jelineks in der Rubrik „Aktuelles“ herausgegeben, jedoch ermöglichte die Gestaltung der Website ${ }^{39}$ und die Publikation der einzelnen Texte nur eine einseitige Kommunikation, denn es gab keine Kommentarfunktion und man verzichtete auf jede andere Art Partizipationsmöglichkeit seitens der Leser. Mehrmals wurde und wird in strenger Form auf die Urheberrechte aufmerksam gemacht. Der fertige Roman wurde dann in Form einer einfachen PDF-Datei auf der Homepage in der Kategorie Prosa jeweils für die entsprechenden Geräte, PCs, Tablets und Smartphones in angepasster Form eingestellt. Dadurch wird die Verwandtschaft des Romantextes mit der alten Medientradition unterstrichen und damit die digitale Literatur anhand einiger aktueller Definitionen, die technologische Merkmale, Intermedialität und Paradigmen der Vernetzungsstrukturen voraussetzten, abgelehnt. ${ }^{40}$ Die Medialität des virtuellen Raumes im Internet als Publikationsform weist einige Paradoxe in der Erscheinungsform auf: auf der einen Seite ist der Inhalt im Internet immer präsent, kann auf der anderen aber jederzeit verschwinden und unzugänglich werden. „Der ausschließlich digital publizierte Privatroman steht daher im Zeichen des Verschwindens der Autorin: Mit der Publikationsform, dem Inhalt und der Ästhetik des Romans zeigt Jelinek ihre paradoxe Existenzform auf, "41 schreibt Lena Lang und deutet neben dem Verschwinden Jelineks aus dem öffentlichen Raum auch auf die Distanzierung der Autorin vom deutschsprachigen Literaturbetrieb hin:

„Durch die Veröffentlichung des Privatromans nimmt die Autorin die Abseitsposition vehement ein und verhandelt auch im Roman ihre Abdrängung. Die Erzählerin wird nicht müde zu betonen, dass ihr Schreiben nur gering geschätzt werde, dass ihr Text für den Leser ungenießbar und unwichtig sei und eignet sich die in der Presse kursierenden Beleidigungen an. [...] Dem Literaturbetrieb, von dem sich Elfriede Jelinek distanziert, stellt sie das Ideal der Demokratisierung und Enthierarchisierung durch die Ort- und Grenzenlosigkeit des Netzes gegenüber und schließt damit an die in den 1990er Jahren geprägten sozialen Utopien an. Dies

38 JELINEK 2008.

39 Wegen der einzelnen Gestaltungselemente der Website vgl. LANG [Anm. 26], S. 4-5.

40 Vgl. hierzu SIMANOWSKI 2002 oder HEIBACH 2003.

41 LANG [Anm. 26], S. 11. 
kann auch eine Erklärung dafür sein, dass sich das Design von Elfriede Jelineks Website seit der Erstellung im Jahr 1996 nicht wesentlich verändert hat und optisch noch an die Frühphase des Internet mit den damaligen Vorstellungen von Anarchie und Antikommerzionalität erinnert." ${ }^{42}$

Durch die Distribution der Texte mittels Website wird der persönliche Charakter der Mitteilung unterstrichen, was zur noch schwierigeren oder sogar unmöglichen Unterscheidung zwischen Autorin und Erzählerin oder Romanfigur führt. Einen Ausweg aus der Kategorisierung Autobiografie und Fiktion ermöglicht auch die Bezeichnung Privatroman im Untertitel, aber viele Signale wie die Initialen der Erzählerin, ihr Alter, der Beruf als Schriftstellerin oder ihr Verhältnis zu Österreich rekurrieren auf sie selbst. So scheint sie den Text auktorial zu beherrschen, aber zugleich mit dem Vorwissen der Leser und mit ihren Erwartungen ein Spiel zu spielen. Trotzdem behält die Erzählerstimme genug Autonomie und spricht sogar die Autorin als Figur an. ${ }^{43}$ Die Postmodernität der Autorin-Erzählerin spiegelt sich auch in der Hauptfigur Brigitte K. wider, die einmal auch als Bestandteil der Erzählerexistenz bezeichnet wird:

„Es können sich immer, wie bei Brigitte, die ein altes, abgelegtes Ego von mir ist, die ich auch schon ziemlich, nein: unziemlich alt bin, überraschende Perspektiven eröffnen, zum Beispiel, daß ich nicht schwimmen kann." 44

Die Hauptfigur und schließlich Mörderin Brigitte K. ist eine Musiklehrerin. Bärbel Lücke weist darauf hin, dass die Figur nicht nur auf die Autorin und Erzählerin des Romans rekurriert.

„Eine Musiktreibende und -lehrende ist z.B. die Doppelgängerin der Erzählerin-Autorin-Chatterin-Sprecherin, Brigitte K. (eine entfernte oder gar nahe Verwandte der Damen K., Mutter und Tochter, aus der Klavierspielerin? “ “45

Als symptomatisch für ein postmodernes Erzählen wirken weiter die von der Erzählerin betonte Unfähigkeit des linearen Erzählens, die Zeitsprünge und Spiele mit dem Rezipienten: „[...] wo bin ich hier eigentlich? Ach ja, im Früher bin ich, aber wollte ich erzählen, müßte ich irgendwie ins Später kommen, was mir sehr schwerfällt [...].“46 Jelinek schafft mit ihrem Roman eine dicht gewebte Montage verschiedenster Zitate und ein Referenzsystem, in dem bewusst traditionelle Strukturen in der Sprache, Narration, Geschichtserzählung (Holocaust), in Vergessen, Erinnerung usw. aufgebrochen werden. Auf der eigenen Homepage bildet die Autorin eine scheinbar private, trotzdem sehr

42 LANG [Anm. 26], S. 12-13.

43 S. Anm. 1.

44 JELINEK [Anm. 21], S. 108.

45 LÜCKE 2007.

46 JELINEK [Anm. 21], S. 151. 
öffentliche Plattform, in der sie, zurückgezogen aus dem öffentlichen Verkehr, wirksam zur Öffentlichkeit sprechen und problematische oder provokante Themen kommentieren kann.

\section{Fazit}

Die Mitgestaltung des Diskurses war eine wirksame Strategie, mit der Thomas Bernhard die Rezeption seines Werkes zu beeinflussen versuchte. Der Autor etablierte sich als eine allgemein bekannte Persönlichkeit, deren Provokation und öffentliche Geste, die die Medien Presse und Fernsehen gern übernahmen und die intensive Reaktionen in der österreichischen Öffentlichkeit hervorriefen, zum Bestandteil der Wirkung seines CEuvres wurden. Daher kann man feststellen, dass die radikalen Überlegungen der Erzähler und Figuren eine Fortsetzung der Stellungnahmen des Schriftstellers und Dramatikers (oder im übertragenen Sinne auch des Großvaters Johannes Freumbichler ${ }^{47}$ ) gewesen sind. Das beweisen nicht nur die zu Lebzeiten Thomas Bernhards herausgegebenen Texte und aufgeführten Dramen, sondern auch die privat geführte Korrespondenz mit dem Verleger und andere nicht redigierte Texte, die erst später aus dem Nachlass des Autors publiziert worden sind. Während Bernhard beim literarischen Feld der Laien nicht immer Verständnis für seine Kommunikation fand, war das internationale Feld der professionellen Vermittler, das sich für die Rezeption auch nach dem Tod des Autors eingesetzt hat. Kurz nach diesem im Jahr 1989 hat sich auch im deutschsprachigen Raum die Computerisierung und Vernetzung durch das Internet massiv verbreitet. Mitte der neunziger Jahre entsteht eine alternative literarische Szene, die zunächst experimentell mit der digitalen Literatur arbeitet. In der Zeit erstellt auch Elfriede Jelinek ihre Website, die sie allmählich als die einzige Publikations- und Kommunikationsplattform genutzt hat. Auch sie (ähnlich wie Bernhard) setzt auf die nicht immer konsequente Unterscheidung zwischen sich als Autorin bzw. Bloggerin und den Erzählerfiguren in ihrer Prosa. Der Akt des Verschwindens wurde zum wichtigen Motiv nicht nur im Internet-Roman „Neid“, sondern auch in der Gestaltung der Website und in der Selbstinszenierung Jelineks durch diese. ${ }^{4}$ So ist auch bei Jelinek das digitale Spiel mit dem Vorwissen und den Erwartungen der Leser in den neuen Medien eine zugewachsene Qualität ${ }^{49}$ ihres Werkes geworden und die kompliziert gelegten Bedeutungs- und Anspielungsschichten des Romans „Neid“ erheben diese Pose auf ein neues Niveau.

47 „Bernhard selbst hat in dem Fernsehmonolog Drei Tage (1971) mit einer vielzitierten Aussage die grundsätzliche Übereinstimmung seiner fiktiven Protagonisten mit Freumbichler behauptet: ,[...] das alles ist in den Büchern später, und diese Figuren, Männerfiguren, das ist immer wieder mein Großvater mütterlicherseits... “ (Zitat im Zitat: FELLINGER 1993, S. 10) MITTERMAYER 2002, S. 14.

48 LANG [Anm. 26], S. 16.

49 Vgl. hierzu Anm. 11. 


\section{Quellen und Literatur}

ANTOS, Gerd [u.a.] (2005) (hrsg.): Wissenstransfer durch Sprache als gesellschaftliches Problem, Frankfurt am Main.

AUER, Johannes [u.a] (2002-2012) (hrsg.): Netzliteratur.net. 2002-2012. http://www.netzliteratur. net (14. 8. 2018).

BARTHES, Roland (2000): Der Tod des Autors. In: JANNIDIS, Fotis [u.a.] (hrsg.): Texte zur Theorie der Autorschaft. Stuttgart (2000), S. 185-197.

BAYER, Wolfram (1995) (hrsg.): Kontinent Bernhard, Wien.

BERNHARD, Thomas (1988): Holzfällen. Eine Erregung. Frankfurt am Main.

BERNHARD, Thomas (1989): Alte Meister. Komödie. Frankfurt am Main.

BERNHARD, Thomas (1992): Auslöschung. Ein Zerfall. Frankfurt am Main.

BERNHARD, Thomas (1995): Heldenplatz. Frankfurt am Main.

BERNHARD, Thomas (2009): Meine Preise. Frankfurt am Main.

BEUTNER, Eduard [u.a.] (hrsg.) (2010): Lesen. Heute. Perspektiven. Innsbruck.

BOURDIEU, Pierre (2001): Die Regeln der Kunst. Genese und Struktur des literarischen Feldes. Frankfurt am Main.

BURGTHEATER WIEN (1989) (hrsg.): Heldenplatz. Eine Dokumentation. Wien.

DERRIDA, Jacques (2003): Die Schrift und die Differenz. Frankfurt am Main.

DITTMAR, Jens (1990): Der Bernhardiner: ein wilder Hund. Tomaten, Satiren und Parodien über Thomas Bernhard, Wien.

FELLINGER, Raimund (1993) (hrsg.): Thomas Bernhard. Ein Lesebuch. Frankfurt am Main.

FELLINGER, Raimund [u.a.] (2009) (hrsg.): Thomas Bernhard - Siegfried Unseld. Der Briefwechsel. Frankfurt am Main.

FOUCAULT, Michel (1997): Archäologie des Wissens, Frankfurt am Main.

FOUCAULT, Michel (2000): Was ist ein Autor? In: JANNIDIS, Fotis [u.a.] (hrsg.): Texte zur Theorie der Autorschaft. Stuttgart, S. 198-232.

FRANZ [u.a.] (2010): Unter den Augen Dritter. Akteur- und Zuschauerkonstellationen im Web 2.0. In: Weimarer Beiträge 56.1 (2010), S. 97-124.

GENETTE, Gérard (2001): Paratexte. Das Buch vom Beiwerk des Buches. Frankfurt am Main.

HEIBACH, Christiane (2003): Literatur im elektronischen Raum. Frankfurt am Main.

HOHENDAHL, Peter Uwe (1985) (hrsg.): Geschichte der deutschen Literaturkritik (1730-1980). Stuttgart.

JANKE, Pia (2002) (hrsg.): Die Nestbeschmutzerin. Jelinek und Österreich. Salzburg.

JELINEK, Elfriede [u.a.] (2004): Habe gebetet, dass ich ihn nicht bekomme. In: Profil, 9. Oktober. http://www.profil.at/home/habe-95051 (14. 8. 2018).

JELINEK, Elfriede (2007-2008): Neid. Privatroman. https://www.elfriedejelinek.com (14. 8. 2018).

JELINEK, Elfriede (2007-2008): Neid. Privatroman. PDF. https://www.elfriedejelinek.com/ NEID.pdf (14. 8. 2018).

JELINEK, Elfriede (2008): Keine Anweisung, keine Auszahlung, kein Betrag, kein Betrug. (Ein paar Anmerkungen zu Neid). https://www.elfriedejelinek.com/fanmerk.htm (14. 8. 2018).

JUNG, Matthias (2001): Unsystematischer Wissenstransfer über die Medien. In: WICHTER, Sigurd [u.a.] (hrsg.): Wissenstransfer zwischen Experten und Laien. Umriss einer Transferwissenschaft. Frankfurt am Main, S. 275-286.

KOPPENSTEINER, Jürgen (1982): Anti-Heimatliteratur in Österreich. Zur literarischen Heimatwelle der siebziger Jahre. (Modern Austrian Literature 15, Nr. 2), S. 1-11. 
LANG, Lena (2016): Elfriede privat?! Elfriede Jelineks digitale Selbstinszenierung. In: Textpraxis 12 (1.2016), http://www.uni-muenster.de/textpraxis/lena-lang-elfriede-jelineks-digitale-selbstinszenierung (14. 8. 2018).

LINK, Kay (2000): Die Welt als Theater - Künstlichkeit und Künstlertum bei Thomas Bernhard. Stuttgart.

LORENZ, Ann-Kathrin (2013): Der Hypertext ist tot! Ein Interview mit der Internet-Literatin Susanne Berkenheger. In: literaturkritik.de 4 (2013). https://literaturkritik.de/public/rezension. php?rez_id=17767\&ausgabe=201304 (14. 8. 2018).

LÜCKE, Bärbel: (Nicht-)Erzählen im Wurmloch der Zeit. Zu Elfriede Jelineks Internetroman Neid. https://jelinetz.com/2007/04/26/barbe-llucke-nicht-erzahlen-im-wurmloch-der-zeit (14.8. 2018).

PECKA, Zdeněk (2010): Thomas Bernhard - ein Begriff ohne Meriten? Zur Stellung Thomas Bernhards in der Kanonbildung der österreichischen Literatur in Tschechien. In: KNAFFL, Arnulf (Hrsg.): Kanon und Literaturgeschichte, Wien, S. 107-116.

MITTERMAYER, Manfred (2002): Der ,Großvater mütterlicherseits‘. In: HUBER, Martin [u.a.] (hrsg.): Thomas Bernhard und seine Lebensmenschen. Der Nachlaß. Frankfurt am Main.

MÜLLER, Alexandra [u.a.] (2013): Die Zukunft des Lesens ist das Nicht-Lesen Neue Medien, neue Literatur? https://www.swr.de/swr2/wissen/neue-medien-neue-literatur/-/id=661224/ $\operatorname{did}=11338128 /$ nid=661224/kj6shm/index.html (14. 8. 2018).

SCHMIDT-DENGLER, Wendelin (1989): Der Übertreibungskünstler. Wien.

SIMANOWSKI, Roberto (2002): Interfictions. Vom Schreiben im Netz. Frankfurt am Main.

SIMANOWSKI, Roberto (2012): Vom neuen Eigenleben der Wörter. Drei Fragen zur digitalen Literatur. http://parapluie.de/archiv/cyberkultur/literatur (14. 8. 2018).

STOCKER, Günther (1994): Ein rebellisches Fossil. Die fiktionale Literatur im Zeitalter der modernen Kommunikationstechnologien. Aachen.

SUTER, Beat (2012): Von Theo Lutz zur Netzliteratur. Die Entwicklung der deutschsprachigen elektronischen Literatur. https://www.netzliteratur.net/suter/Geschichte_der_deutschsprachigen_Netzliteratur.pdf (14. 8. 2018).

TRILCKE, Peer (2013): Ideen zu einer Literatursoziologie des Internets. Mit einer Blogotop-Analyse. In: Textpraxis 7 (2.2013). http:/ / www.uni-muenster.de/textpraxis/ peer-trilcke-literatursoziologie-des-internets (14. 8. 2018).

Dr. phil. Zdeněk Pecka / pecka@pf.jcu.cz

Jihočeská univerzita v Českých Budějovicích

Katedra germanistiky, Pedagogická fakulta

Jeronýmova 10, 37115 České Budějovice, CZ 